\title{
Obtenção e caracterização de carvão ativado de caroço de buriti (Mauritia flexuosa L. f.) para a avaliação do processo de adsorção de cobre (II)
}

\begin{abstract}
Marcos Vinicios de Souza PINTO ${ }^{1}$, Denilson Luz da SILVA¹, Augusto César Fonseca SARAIVA²
RESUMO

$\mathrm{Na}$ região amazônica, algumas indústrias despejam cobre nos corpos receptores que, em elevadas concentrações, é tóxico para os seres vivos. A remoçẫo de cobre de efluentes industriais é realizada por diversos processos como a adsorçấo. Neste trabalho mostrou-se o resultado da adsorção de cobre (II) em carvão ativado de caroço de buriti carbonizado a $400^{\circ} \mathrm{C}$ e ativado a 900 ${ }^{\circ} \mathrm{C}$. O carvão ativado foi caracterizado em termos de área específica, tamanho dos poros, densidades aparente e real, porosidade, microscopia eletrônica de varredura, conteúdo de cinzas, $\mathrm{pH}$, umidade, carbono fixo e grupos funcionais de superfície. O estudo de equilíbrio de adsorção avaliou a influência do diâmetro das partículas do carvão, do tempo de contato adsorvente/adsorbato, do $\mathrm{pH}$ e da concentração inicial da solução de cobre (II) sobre a remoção de cobre (II). Com base nos resultados, concluiu-se que há uma maior eficiência de remoção de cobre (II) para diâmetro $<0,595 \mathrm{~mm}$, tempo de contato de 300 minutos, $\mathrm{pH}$ 4,0 e concentraçóes iniciais de cobre (II) de 50 e $80 \mathrm{mg} \mathrm{L}^{-1}$. O modelo matemático de Langmuir foi o que melhor se ajustou aos dados de equilíbrio de adsorção. A partir do tempo de contato de 15 minutos todas as concentraçóes de equilíbrio ficaram abaixo do máximo permitido de $1,0 \mathrm{mg} \mathrm{L}^{-1}$ previsto pela legislação vigente para lançamento de efluentes em corpos receptores.
\end{abstract}

PALAVRAS-CHAVE: Adsorção, Cu (II), Carvão Ativado, Buriti.

\section{Production and characterization of the activated carbon from buriti stone (Mauritia flexuosa L. f.) to evaluate the adsorption's process of copper (II)}

\section{ABSTRACT}

In the Amazon region some industries discharges copper into watercourse that in high concentrations is toxic to the biota. The removal of copper from industrial effluent is performed by several processes such as adsorption. This work shows the result of copper (II) adsorption on activated carbon obtained from buriti kernel, carbonized at $400{ }^{\circ} \mathrm{C}$ and activated at $900{ }^{\circ} \mathrm{C}$. The activated carbon was characterized according to specific area, pore size, apparent and real density, porosity, scanning electron microscopy, ash content, $\mathrm{pH}$, moisture, fixed carbon and surface functional groups. The study of adsorption equilibrium evaluated the influence of the coal particles diameter, contact time adsorbent/adsorbate, $\mathrm{pH}$ and copper (II) solution initial concentration on copper (II) remotion. The results showed a higher removal efficiency of copper (II) to the diameter D < $0.595 \mathrm{~mm}$, contact time of 300 minutes, $\mathrm{pH}$ of 4.01 and the copper (II) initial concentrations of 50 and $80 \mathrm{mg}$ L-1. The mathematical model of Langmuir was the best fit to the adsorption equilibrium data. From the contact time of 15 minutes, all the equilibrium concentrations were below the allowed maximum of 1,0 mg L-1 provided by law for discharging effluents into receiving bodies.

KEYWORDS: Adsorption, Copper (II), Activated Carbon, Buriti. 


\section{INTRODUÇÃO}

A poluição do meio ambiente tornou-se assunto de interesse público em todas as partes do mundo. Náo somente os países desenvolvidos vem sendo afetados pelos problemas ambientais, como também as naçóes em desenvolvimento sofrem com os grandes impactos da poluição. Isso decorre de um rápido crescimento econômico associado à exploração de recursos naturais. Ao lado dos crescentes problemas provocados pela contaminação do meio ambiente, estão os processos utilizados para extrair matérias-primas e para transformá-las em produtos para fins de consumo em elevada escala (Braile e Cavalcante 1993).

A partir do desenvolvimento industrial e das atividades ligadas à indústria, o volume de resíduos industriais e pósconsumo despejados no meio ambiente aumentou de modo expressivo, superando a capacidade do ambiente de promover sua autodepuração. Diante dessa situação, torna-se necessário um controle rígido dos resíduos sólidos e efluentes industriais, a fim de se evitar a deterioração da qualidade de vida das populaçôes ao redor dos centros industriais (Souza 2009).

Atualmente os poluentes que causam maiores preocupaçóes ambientais são os metais pesados, que são produzidos e descartados pelas diferentes atividades tecnológicas. Alguns metais pesados são substâncias altamente tóxicas e não são compatíveis com a maioria dos tratamentos biológicos de efluentes existentes. Devido aos efeitos tóxicos provocados por esses metais nos seres vivos, existe um significativo interesse no desenvolvimento e aperfeiçoamento das técnicas utilizadas na sua remoção (Aguiar e Novaes 2002).

Os compostos de cobre bivalente ou cobre (II), são mais tóxicos, visto que as valências (I) e (III) estão geralmente na forma complexada ou insolúvel (Lee 1980). Se por um lado a deficiência de cobre no organismo humano pode ocasionar anemia, por outro a exposiçáo oral a níveis elevados pode causar vômito, diarréia, cólica estomacal e náusea (Bryan e Langston 1992).

Os compostos de cobre (II) são introduzidos no ambiente, a partir de inúmeros processos industriais como: indústria de papel e celulose, indústria de fertilizantes, refinarias de petróleo, produção de aço e na indústria de metais não ferrosos, dentre outros (Ramos 2005). Vários métodos de tratamento de efluentes industriais contaminados por uma elevada concentração de metal pesado são estudados, tais como: precipitação, filtração por membrana/osmose, eletrodeposição, troca iônica, oxidação/redução e adsorção, sendo a adsorção a operação de separação mais efetiva no tratamento de águas residuárias (Liu et al. 2007).

Diversas pesquisas são realizadas com o objetivo de se utilizar uma ampla gama de adsorventes como fonte alternativa no tratamento de águas residuárias, tendo a finalidade de minimizar ao máximo permissível a concentração de íons indesejáveis no ambiente aquático (Kaikake et al. 2007). Desta maneira, torna-se ambientalmente viável o uso de alternativas para outros resíduos de biomassa, tais como: caroço de açaí, caroço de buriti, casca de coco e, casca de castanha-do-pará, dentre outros. (Nunes et al. 2009).

Um dos efetivos usos dos resíduos de biomassa proveniente da agricultura é a produção de carvão ativado por conversão termo-química. Muitas revisóes neste assunto sáo avaliadas, com aplicaçôes em remoção de metais pesados, compostos orgânicos e corantes (Crini 2006). Os resíduos da agricultura são os mais comuns materiais brutos estudados para este fim, visto que eles são renováveis, usualmente acessíveis em larga quantidade e de custo mais baixo do que outros materiais para manufaturar uma diversidade de tipos de adsorvente (Ajmal et al. 2005; Tan et al. 2007; Karagöz et al. 2008).

O buriti (Mauritia flexuosa L. f.), que cresce espontaneamente nas várzeas do Brasil Central, nos terrenos pantanosos próximos de cursos d'água permanente, no alto de serras e principalmente na região amazônica, é muito utilizado na culinária na forma de geléia, sorvetes, cremes e doces, além de óleo comestível (Manhães 2007).

Atualmente muitas pesquisas estão voltadas para a obtenção de biodiesel a partir do óleo extraído da casca e da polpa desse fruto, devido a problemas com o aquecimento global, decaimento das reservas de combustíveis fósseis e a necessidade urgente de combustíveis renováveis. Contudo, os caroços desse fruto tornam-se um rejeito comum que pode ser reaproveitado como carvão ativado para a remoção de metais pesados em águas residuárias.

Em face do exposto, a adsorção pode se constituir em uma alternativa tecnológica extremamente importante, principalmente pela possibilidade do uso de adsorventes de baixo custo, tal como o carváo ativado do caroço de buriti (CACB), como um processo viável no controle de poluiçáo hídrica. $\mathrm{O}$ processo de adsorção também visa a remoção de cobre (II) para valores adequados à legislação vigente, que no Brasil é a resolução no 430/2011, estabelecida pelo Conselho Nacional de Meio Ambiente (CONAMA), que determina o valor máximo permitido igual a $1,0 \mathrm{mg} \mathrm{L}^{-1}$ de $\mathrm{Cu}$ dissolvido para o lançamento de efluentes em corpos receptores (Brasil 2011).

Neste trabalho, utilizou-se o caroço de buriti como matéria-prima na produção de carvão ativado. A referida matéria-prima foi obtida do rejeito da produção artesanal da extração de óleo do fruto.

O objetivo principal deste trabalho é estudar a adsorção em sistema batelada e com agitação de uma solução de cobre (II) por carvão ativado de caroço de buriti (CACB). O CACB foi produzido por carbonização a $400{ }^{\circ} \mathrm{C}$ e posterior ativação 
física a 900 C. Avaliou-se também a eficiência de remoção de cobre (II) com variaçóes na granulometria, no $\mathrm{pH}$, no tempo de agitação e concentração da solução de cobre (II).

\section{MATERIAL E MÉTODOS}

\section{Obtenção do adsorvente}

Caroços de buriti (CB) foram utilizados como matériaprima na produção de carvão ativado. Essa matéria-prima foi obtida do rejeito da produção artesanal de óleo extraído da polpa. O material foi coletado na Ilha das Onças pertencente à Região Metropolitana de Belém (região das ilhas). As amostras de CB foram carbonizadas, para análise em duplicata, em dois frascos cerâmicos em uma mufla elétrica da marca QUIMIS modelo Q 318M24 - Brasil, requerendo um tempo médio de 25 minutos, com uma velocidade média de aquecimento de $17{ }^{\circ} \mathrm{C}$ por minuto, até estabilização a $400^{\circ} \mathrm{C}$. Uma tampa cerâmica quadrada foi utilizada em cada frasco, deixando um espaço de $1 \mathrm{~mm}$ em cada face para facilitar a saída dos voláteis. O tempo da carbonização foi de três horas. Após esse período, efetuou-se um arrefecimento natural em dessecadores. Testes de rendimento em carvão ( $\mathrm{RC}$ ) e do conteúdo de materiais voláteis do carvão (MVC) para o carvão do caroço de buriti (CCB) foram feitos de acordo com Ramos (2005). Após as análises, $\mathrm{O} \mathrm{CCB}$ contido em um dos frascos foi ativado por um período de uma hora a $800{ }^{\circ} \mathrm{C}$, e o contido no outro frasco, ativado no mesmo período a $900^{\circ} \mathrm{C}$, no mesmo forno e com a mesma velocidade de aquecimento da carbonização.

\section{Ensaio de adsorção para o CACB a $800^{\circ} \mathrm{C}$ e $900^{\circ} \mathrm{C}$}

Um ensaio preliminar foi executado para se decidir qual a melhor temperatura de ativação para os $\mathrm{CACBs}$ obtidos. $\mathrm{O}$ CACB a $900{ }^{\circ} \mathrm{C}$ foi o escolhido, sendo produzido e utilizado nos ensaios posteriores. Este ensaio preliminar consistiu em se fazer um teste de adsorção para as temperaturas de ativação de $800{ }^{\circ} \mathrm{C}$ e $900{ }^{\circ} \mathrm{C}$, mantendo-se constantes os seguintes parâmetros: concentração do adsorvato sulfato de cobre (II) (50 mg L ${ }^{-1}$, obtido de uma solução estoque a $1000 \mathrm{mg} \mathrm{L}^{-1}$ de sulfato cúprico, preparada do mesmo sal); quantidade de adsorvente (1,0 g por $100 \mathrm{~mL}$ de adsorvato); efeito do tamanho das partículas $(0,595 \mathrm{~mm}<\mathrm{D}<1,19 \mathrm{~mm}$, partículas estas que foram classificadas após 15 minutos de agitação em máquina vibratória modelo RO-TAP da marca Produtest no404 - Alemanha); $\mathrm{pH}$ ( $\mathrm{pH}$ natural da solução, $\mathrm{pH}=5,18$ ); temperatura (temperatura ambiente, $\mathrm{T}=28^{\circ} \mathrm{C}$ ) e o tempo de contato da solução de sulfato de cobre (II) com o adsorvente (60 minutos com uma freqüência de agitação de $150 \mathrm{rpm}$ ). Para este ensaio as amostras de CACB foram previamente secas por uma hora em estufa de secagem e esterilização modelo 315 SE da marca FANEM - Brasil na temperatura de $150 \pm 1{ }^{\circ} \mathrm{C}$. Após o período de agitação na mesa agitadora modelo CERTOMAT ${ }^{\circ} \mathrm{MO}$ da marca B. BRAUN BIOTEC internacional - USA as amostras foram filtradas, preservadas com ácido nítrico p.a, para baixar o $\mathrm{pH}$, e acondicionadas em frascos vítreos com tampa plástica para posterior análise em absorção atômica modelo SPECTRAA 50 da marca Varian USA. Os resultados da absorção atômica indicaram melhor eficiência de remoção para o CACB a $900{ }^{\circ} \mathrm{C}(97,35 \%)$, o qual foi produzido para os posteriores ensaios de adsorção.

\section{Produção e caracterização do CACB a $900^{\circ} \mathrm{C}$}

Dez bateladas foram realizadas para a obtenção de CACB a $900{ }^{\circ} \mathrm{C}$ em um forno mufla. Cada batelada foi feita com a utilização de dois recipientes cerâmicos. O cálculo do rendimento médio em carvão ativado (RMCA) e do material volátil do carvão ativado (MVCA) foi realizado de acordo com Ramos (2005). O CACB foi caracterizado quanto a: área específica (BET) no equipamento da marca Micromeritics modelo ASAP 2020 V3 2H - USA, no laboratório de metais/ cerâmica do Centro de Caracterização e Desenvolvimento de Materiais (CCDM) da Universidade Federal de São Carlos (UFSCar); distribuição dos poros através do porosímetro de mercúrio da marca Aminco, modelo 5000 psi - USA, no laboratório de metais/cerâmica do CCDM da UFSCar; densidade aparente pelo método ASTM D 2854-09; densidade real pelo equipamento AccuPyc 1330, no de série 2399, marca Micromeritics - USA; porosidade pelo método da ABNT. NBR 9165, 1985; microscopia eletrônica de varredura em microscópio, modelo LEO-1430 com corrente do feixe de elétrons $(90 \mu \mathrm{A})$, voltagem constante $(10 \mathrm{kV})$, distância de trabalho (15-12 mm), sendo as amostras preparadas com uma fina camada de ouro usando um metalizador Emitech K550; conteúdo de cinzas pelo método ASTM D 2866-94; pH pelo método ASTM D 3838-05; umidade pelo método ASTM D 2867-04; carbono fixo de acordo com Ramos (2005) e grupos funcionais de superfície determinados de acordo com a metodologia de Boehm (Boehm 1994; Beck et al. 2002; Boehm 2002; Zhao et al. 2005).

\section{Influência do diâmetro das partículas de CACB na adsorção de cobre (II)}

Realizaram-se os ensaios em duplicata em uma mesa agitadora, variando-se os diâmetros de acordo com os seguintes intervalos $(\mathrm{D}>1,19 \mathrm{~mm}) ;(0,595<\mathrm{D}<1,19 \mathrm{~mm})$ e $(\mathrm{D}<$ $0,595 \mathrm{~mm}$ ). Obteve-se esta faixa granulométrica com o uso de peneiras para análise granulométrica da BERTEL em máquina vibratória. Mantiveram-se os seguintes parâmetros constantes: concentração do adsorvato $\left(50 \mathrm{mg} \mathrm{L}^{-1}\right.$ de cobre (II)), $\mathrm{pH}$ inicial da solução $(\mathrm{pH}=5,18)$, tempo de contato adsorvente/adsorvato ( $\mathrm{t}=60$ minutos), frequência de agitação ( $\mathrm{f}=150 \mathrm{rpm}$ ) e quantidade de adsorvente $1 \mathrm{~g}$ de $\mathrm{CACB}$ por $100 \mathrm{~mL}$ de solução de cobre (II). Após o referido tempo as amostras foram filtradas em papel de filtro qualitativo (Frama), preservadas com $\mathrm{HNO}_{3}$ p.a e armazenadas a baixa temperatura para posterior análise em absorção atômica. 


\section{Influência do tempo de contato adsorvente/ adsorvato na adsorção de cobre (II)}

Realizaram-se os ensaios em duplicata na mesma mesa agitadora nos tempos de agitação de 15, 30, 60, 120, 180, 240 e 300 minutos. Mantiveram-se os seguintes parâmetros constantes: diâmetro do CACB $(0,595<\mathrm{D}<1,19 \mathrm{~mm})$, concentração do adsorvato $\left(50 \mathrm{mg} \mathrm{L}^{-1}\right.$ de cobre (II)), $\mathrm{pH}$ inicial da solução $(\mathrm{pH}=5,18)$, frequência de agitação $(\mathrm{f}=$ $150 \mathrm{rpm}$ ) e quantidade de adsorvente $1 \mathrm{~g}$ de CACB por 100 $\mathrm{mL}$ de soluçẫo de cobre (II).

\section{Influência do pH na adsorção de cobre (II)}

Realizaram-se os ensaios em duplicata em uma mesa agitadora, variando-se os valores de $\mathrm{pH}$ inicial em 3,0; 4,01; 5,18 , que é o pH natural da solução de cobre (II) e 6,0. Esses valores foram adquiridos pela adição de $\mathrm{HCl}\left(0,1 \mathrm{~mol} \mathrm{~L}^{-1}\right)$ ou $\mathrm{NaOH}\left(0,1 \mathrm{~mol} \mathrm{~L}^{-1}\right)$ e medidos em um potenciômetro de bancada modelo $\mathrm{pH} 21$, marca HANNA - Brasil. Mantiveram-se os seguintes parâmetros constantes: diâmetro do $\mathrm{CACB}(0,595<\mathrm{D}<1,19 \mathrm{~mm})$, concentraçáo do adsorvato (50 $\mathrm{mg} \mathrm{L}^{-1}$ de cobre (II)), tempo de contato adsorvente/ adsorvato $(\mathrm{t}=60 \mathrm{~min}$.), frequência de agitação $(\mathrm{f}=150 \mathrm{rpm})$ e quantidade de adsorvente $1 \mathrm{~g}$ de CACB por $100 \mathrm{~mL}$ de solução de cobre (II).

\section{Equilíbrio de adsorção}

Tendo-se conhecimento dos melhores resultados da influência dos parâmetros físicos e físico-químicos na adsorção, efetuou-se o ensaio de adsorção. O ensaio foi realizado em duplicata em uma mesa agitadora com as seguintes concentraçôes de cobre (II): 5, 10, 30, 50, $100 \mathrm{e}$ $200 \mathrm{mg} \mathrm{L}^{-1}$. Estas concentrações foram obtidas da solução estoque a $1000 \mathrm{mg} \mathrm{L}^{-1}$ do mesmo sal previamente preparada. Utilizou-se o volume de $100 \mathrm{~mL}$ para cada concentração. Os parâmetros diâmetro do CACB $(0,595<\mathrm{D}<1,19 \mathrm{~mm})$; $\mathrm{pH}$ inicial da solução ( $\mathrm{pH}$ da amostra); tempo de contato (60 minutos); frequência de agitação (150 rpm) e concentração do adsorvente ( $1 \mathrm{~g}$ por $100 \mathrm{~mL}$ de solução) permaneceram constantes.

Em todos os ensaios acima descritos, após o referido tempo de agitaçâo, foram feitos os mesmos procedimentos de filtragem, preservação e armazenagem das amostras para posterior análise em absorção atômica.

\section{Ajuste aos modelos matemáticos de equilíbrio de adsorção}

Os modelos matemáticos existentes mais utilizados para sistema sólido-líquido na produção de dados de equilíbrio são os modelos de Langmuir e Freundlich. Por essa razão eles foram testados no estudo da adsorção de uma solução de cobre (II). Uma regressão linear foi feita no software Microcal Origin Pro 7.0 para avaliar o melhor modelo de equilíbrio. As equaçôes do modelo de Langmuir são mostradas a seguir (Moreno-Castilha 2004).

$$
\mathrm{q}_{\mathrm{e}}=\frac{\mathrm{q}_{\text {máx }} \cdot \mathrm{b} \cdot \mathrm{C}_{\mathrm{e}}}{1+\mathrm{b} \cdot \mathrm{C}_{\mathrm{e}}} \quad \text { (forma não linear) }
$$

$\mathrm{Ou}$

$$
\frac{\mathrm{C}_{\mathrm{e}}}{\mathrm{q}_{\mathrm{e}}}=\frac{1}{\mathrm{q}_{\text {máx }} \cdot \mathrm{b}}+\frac{1}{\mathrm{q}_{\text {máx }}} \cdot \mathrm{C}_{\mathrm{e}} \quad \text { (forma linear) }
$$

Onde $\left(\mathrm{q}_{\mathrm{e}}\right)$ é a massa do adsorvato adsorvida por unidade de massa do adsorvente ( $\mathrm{mg}$ de adsorvente por $\mathrm{g}$ de carvão ativado); ( $\mathrm{C}_{\mathrm{e}}$ ) é a concentração no equilíbrio do adsorvato em solução depois da adsorção $\left(\mathrm{mg} \mathrm{L}^{-1}\right) ;\left(\mathrm{q}_{\text {max }}\right)$ é uma constante empírica que indica a capacidade de adsorçấo na monocamada ( $\left.\mathrm{mg} \mathrm{g}^{-1}\right)$; (b) é uma constante relacionada à energia livre de adsorção ou a constante de equilíbrio de adsorção, sendo o recíproco da concentração na qual a meia saturação do adsorvente é alcançada.

Uma característica essencial da isoterma de Langmuir pode ser explicada em termos do parâmetro $\left(\mathrm{R}_{\mathrm{L}}\right)$ denominado fator de separação, definido pela equação 3 , que possibilita avaliar a forma da isoterma: isoterma irreversível $\left(R_{L}=0\right)$; favorável $\left(0<\mathrm{R}_{\mathrm{L}}<1\right)$; linear $\left(\mathrm{R}_{\mathrm{L}}=1\right)$ e desfavorável $\left(\mathrm{R}_{\mathrm{L}}>0\right)$. $\mathrm{R}_{\mathrm{L}}$ indica a eficiência do adsorvente sob estudo e é adimensional, b é a constante de Langmuir e $\mathrm{C}_{\mathrm{i}}$ é a concentração do metal em solução (mg L-1 $)$ (Kadirvelu et al. 2001).

$$
\mathrm{R}_{\mathrm{L}}=\frac{1}{1+\mathrm{bC}_{\mathrm{i}}}
$$

As equaçóes do modelo de Freundlich são apresentadas a seguir (Moreno-Castilha 2004):

$$
\mathrm{q}_{\mathrm{e}}=\mathrm{K}_{\mathrm{ad}} \cdot \mathrm{C}_{\mathrm{e}}^{1 / \mathrm{n}} \quad \text { (forma âo linear) }
$$

$$
\begin{aligned}
& \text { Ou } \\
& \log \left(\mathrm{q}_{\mathrm{e}}\right)=\log \left(\mathrm{K}_{\mathrm{ad}}\right)+\frac{1}{\mathrm{n}} \cdot \log \left(\mathrm{C}_{\mathrm{e}}\right) \text { (forma linear) }
\end{aligned}
$$

Onde $\left(\mathrm{q}_{\mathrm{e}}\right)$ é a quantidade de adsorvato por unidade de adsorvente $\left(\mathrm{mg} \mathrm{g}^{-1}\right.$ ou mol g $\left.{ }^{-1}\right) ;\left(\mathrm{C}_{\mathrm{e}}\right)$ é a concentração do adsorvato no equilíbrio $\left(\mathrm{mg} \mathrm{L}^{-1}\right.$ ou $\left.\mathrm{mol} \mathrm{L}^{-1}\right) ;\left(\mathrm{K}_{\mathrm{ad}}\right)$ está relacionado à capacidade de adsorçáo do adsorvato pelo adsorvente [(mg g-1) $\left.(\mathrm{L} \mathrm{mg-1})^{1 / \mathrm{n}}\right]$; e (n) depende das características da adsorção (adimensional). ( $\mathrm{K}_{\mathrm{ad}}$ e n) são coeficientes que são determinados empiricamente através de regressão linear. 


\section{RESULTADOS E DISCUSSÃO}

Caracterização do CACB a $900{ }^{\circ} \mathrm{C}$

$\mathrm{Na}$ Tabela 1 são apresentados os resultados da caracterização do CACB. Na Figura 1 é apresentada a microscopia eletrônica de varredura do CACB. A foto comprova uma estrutura porosa com consequente elevação da área superficial do CACB, porém muito inferior a de outros carvôes ativados, de 200 a $1500 \mathrm{~m}^{2} \mathrm{~g}^{-1}$ (Di Bernardo e Dantas 2005). Os resultados da porosimetria indicam que $80 \%$ do tamanho dos poros do carvão são classificados como macro poros, o que poderia ocasionar uma baixa capacidade de adsorção. Contudo, os valores das propriedades físicas e físico-químicas do $\mathrm{CACB}$ contribuíram para a elevada adsorção de uma solução de cobre (II). Isso se torna evidente pelo valor do $\mathrm{pH}$ do $\mathrm{CACB}$ acima de 10, que segundo Di Bernardo e Dantas (2005) o pH deve estar abaixo de 4 ou acima de 10 para que o carváo adsorva muitos elementos, logo o $\mathrm{pH}$ alcalino do CACB o inclui nestas zonas o que facilita a adsorção de muitos elementos. Também é perceptível que a presença de grupos carboxílicos em concentraçóes elevadas melhora significativamente o processo de adsorção quando o $\mathrm{pH}$ de trabalho da solução de cobre (II) está na faixa ácida ( $\mathrm{pH}$ natural da amostra de 5,18). Os grupos carboxílicos são encontrados em maior quantidade, em comparação com os grupos hidroxil fenólicos e grupos lactonas. Estes dois últimos grupos são considerados relativamente fracos e se dissociam em valores de $\mathrm{pH}$ mais elevados que os grupos carboxílicos (Liu et al. 2007).

Tabela 1 - Caracterização do carvão do caroço de buriti ativado a $900{ }^{\circ} \mathrm{C}$

\begin{tabular}{|c|c|c|}
\hline Propriedades & Resultados & Desvio \\
\hline Rendimento em carvão (\%) & 73,01 & 2,0789 \\
\hline Material volátil do carvão (\%) & 26,99 & 2,0789 \\
\hline Rendimento médio em carvão ativado (\%) & 22,32 & 0,9937 \\
\hline Material volátil do carvão ativado (\%) & 77,68 & 0,9937 \\
\hline Área específica $\left(\mathrm{m}^{2} \mathrm{~g}^{-1}\right)$ & 26,363 & $(*)$ \\
\hline $\begin{array}{l}\text { Distribuição dos poros (Porosimetria): } \\
\text { Diâmetro médio dos poros }(\mu \mathrm{m}) \\
80 \% \text { dos poros entre }(\mu \mathrm{m})\end{array}$ & $\begin{array}{c}2,24 \\
0,83-7,29\end{array}$ & $\begin{array}{l}(*) \\
(*)\end{array}$ \\
\hline Densidade aparente $\left(\mathrm{g} \mathrm{cm}^{-3}\right)$ & 0,8170 & 0,0067 \\
\hline Densidade real $\left(\mathrm{g} \mathrm{cm}^{-3}\right)$ & 1,7594 & 0,0036 \\
\hline Porosidade (\%) & 53,56 & 0,3791 \\
\hline Conteúdo de cinza total (\%) & 8,416 & 0,5163 \\
\hline $\mathrm{pH}$ & 10,70 & 0,0707 \\
\hline Umidade (\%) & 3,72 & 0,2970 \\
\hline Carbono fixo (\%) & 13,904 & 0,9937 \\
\hline $\begin{array}{l}\text { Grupos funcionais de superfície (\%) } \\
-\mathrm{COOH} \\
-\mathrm{COOR} \\
-\mathrm{OH}\end{array}$ & $\begin{array}{l}66,51 \\
18,06 \\
15,43\end{array}$ & $\begin{array}{l}0,0090 \\
0,0221 \\
0,0266\end{array}$ \\
\hline
\end{tabular}

${ }^{*}$ ) Não fornecido pelo Centro de Caracterização e Desenvolvimento de Materiais da Universidade Federal de São Carlos (UFSCAR).

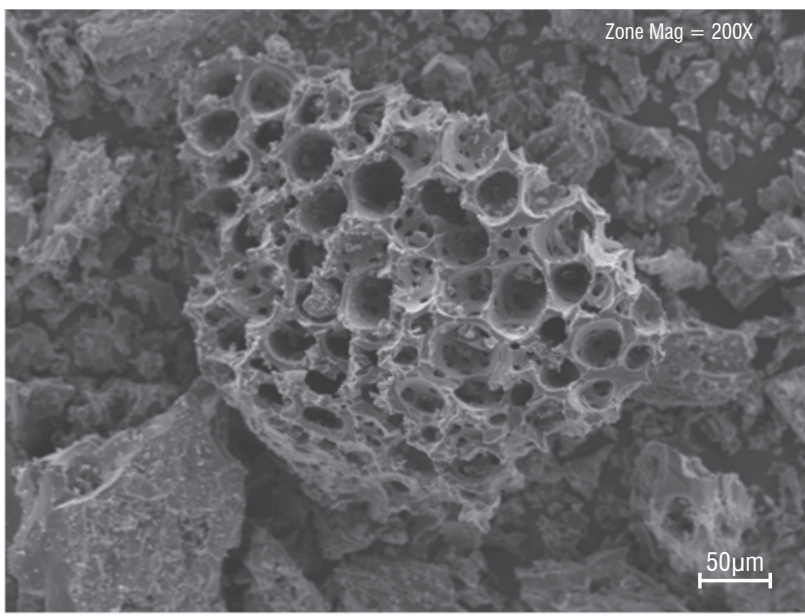

Figura 1 - Imagem do carvão do caroço de buriti ativado a $900^{\circ} \mathrm{C}$, obtida por microscopia eletrônica de varredura

\section{Influência do diâmetro das partículas de CACB na adsorção de cobre (II)}

$\mathrm{Na}$ Tabela 2 são apresentados os resultados obtidos para a influência do diâmetro das partículas do $\mathrm{CACB}$ a $900^{\circ} \mathrm{C}$ na adsorção de cobre (II). Percebe-se que as eficiências de remoção para as três faixas granulométricas são bastante elevadas e bem próximas umas das outras. A eficiência de remoção de cobre (II) aumentou com o decréscimo da faixa granulométrica das partículas, devido ao correspondente aumento da área superficial e melhor acessibilidade aos poros (Nunes et al. 2009). Segundo Senthilkumaar et al. (2005), a quebra das partículas grandes em unidades menores pode também abrir minúsculos canais fechados, os quais podem tornar-se viáveis para a adsorção. Resultados similares no efeito do tamanho das partículas foram relatados para outros resíduos, tais como caroços de fruta (Banat et al. 2003), casca de coco e fibras de juta (Singh et al. 2003; Senthilkumaar et al. 2005) . Como as eficiências estão bastante próximas, escolheu-se a faixa intermediária $(0,595<\mathrm{D}<1,19 \mathrm{~mm})$ como base para o ensaio de equilíbrio de adsorçáo, visto que a referida faixa assemelha-se com a granulometria dos carvóes comerciais.

Tabela 2 - Influência do diâmetro das partículas do carvão ativado do caroço de buriti (CACB) na adsorção de cobre (II)

\begin{tabular}{lccccc}
\hline $\begin{array}{l}\text { Diâmetro do } \\
\text { CACB }(\mathrm{mm})\end{array}$ & $\begin{array}{c}\mathrm{C}_{\mathrm{e}}{ }^{(1)} \\
\left(\mathrm{mg} \mathrm{L}^{-1}\right)\end{array}$ & $\begin{array}{c}\text { Desvio } \\
\text { (95\% de } \\
\text { Confiança) })\end{array}$ & $\begin{array}{c}\text { Intervalo de } \\
\text { Confiança }\end{array}$ & $\begin{array}{c}\mathrm{q}_{\mathrm{e}}{ }^{(2)} \\
\left(\mathrm{mg} \mathrm{g}^{-1}\right)\end{array}$ & $\begin{array}{c}\text { Eficiência } \\
\text { de Remoção } \\
(\%)\end{array}$ \\
\hline $\mathrm{D}>1,19$ & 0,229 & 0,0877 & {$[-0,5588 ; 0,168]$} & 4,9771 & 99,54 \\
$0,595<\mathrm{D}<1,19$ & 0,223 & 0,2121 & {$[0,0324 ; 0,4136]$} & 4,9777 & 99,55 \\
$D<0,595$ & 0,188 & 0,0198 & {$[0,0101 ; 0,3659]$} & 4,9812 & 99,62 \\
\hline $\begin{array}{l}\text { (1) Ce: Concentração de equilíbrio } \\
\text { (') qe: Capacidade de adsorção }\end{array}$ & & & \\
\hline
\end{tabular}




\section{Influência do tempo de contato adsorvente/ adsorvato na adsorção de cobre (II)}

$\mathrm{Na}$ Tabela 3 são apresentados os resultados obtidos para a influência do tempo de contato adsorvente/adsorvato do $\mathrm{CACB}$ a $900^{\circ} \mathrm{C}$ na adsorção de cobre (II). Percebe-se que as eficiências de remoção para os sete tempos de agitaçáo (tempo de contato) são bastante elevadas e bem próximas umas das outras. A eficiência de remoção de cobre (II) aumentou com o aumento do tempo de contato adsorvente/adsorvato, devido haver maior eficiência nas etapas de transporte de massa do processo de adsorção ao longo do tempo (Bansal e Goyal 2005). Observa-se que o tempo inicial de 15 minutos já apresenta uma eficiência de remoção acima de $99 \%$, o que caracteriza que o sistema atingiu o equilíbrio após esse tempo de adsorção. Ao final da adsorção (300 minutos), obteve-se a máxima remoção $(99,64 \%)$ de cobre (II), sendo a concentração de equilíbrio igual a $0,1820 \mathrm{mg} \mathrm{L}^{-1}$. De acordo também com os resultados, temperatura ambiente de $28^{\circ} \mathrm{C}, \mathrm{pH}$ de 5,18 , concentração inicial de cobre (II) de $50 \mathrm{mg} \mathrm{L}^{-1}$, diâmetro < $0,595 \mathrm{~mm}$, frequência de agitação de $150 \mathrm{rpm}$ e quantidade do adsorvente de $1 \mathrm{~g}$ de CACB por $100 \mathrm{~mL}$ de solução do metal, conclui-se que a adsorçáo pode ser descrita por um comportamento cinético em dois estágios, com uma etapa rápida de adsorção inicial durante os primeiros 15 minutos, seguida posteriormente, por uma etapa muito lenta. A rápida adsorçấo de cobre (II) durante os primeiros 15 minutos é um indicativo de que a adsorção ocorre facilmente na superfície do CACB. Os valores das concentrações de equilíbrio (Ce) de cobre (II) obtidos, evidenciam que, a partir do tempo de contato de 15 minutos, as concentraçôes permaneceram abaixo do valor máximo permitido $\left(1,0 \mathrm{mg} \mathrm{L}^{-1}\right)$ preconizado pela legislação nacional, Resolução CONAMA no 430/2011, para o lançamento de efluentes em corpos receptores. Devido às eficiências de remoção serem bastante próximas, escolheu-se o tempo de agitação de 60 minutos para o ensaio de equilíbrio de adsorção, pois não se justificaria um maior consumo de energia para o processo realizado no período de 300 minutos.

Tabela 3 - Influência do tempo de contato (t) entre o carvão ativado do caroço de buriti e uma solução de cobre (II) no processo de adsorção

\begin{tabular}{cccccc}
\hline $\begin{array}{c}\mathrm{t} \\
\text { (min.) }\end{array}$ & $\begin{array}{c}\mathrm{C}_{\mathrm{e}}{ }^{(1)} \\
\left(\mathrm{mg} \mathrm{L}^{-1}\right)\end{array}$ & $\begin{array}{c}\mathrm{q}_{\mathrm{e}}{ }^{(2)} \\
\left(\mathrm{mg} \mathrm{g}^{-1}\right)\end{array}$ & $\begin{array}{c}\text { Desvio } \\
(95 \% \mathrm{de} \\
\text { confiança) }\end{array}$ & $\begin{array}{c}\text { Intervalo } \\
\text { de Confiança }\end{array}$ & $\begin{array}{c}\text { Eficiência } \\
\text { de Remoção } \\
(\%)\end{array}$ \\
\hline 15 & 0,4380 & 4,9562 & 0,0572 & {$[-0,1171 ; 0,9121]$} & 99,12 \\
30 & 0,4375 & 4,9563 & 0,0361 & {$[0,1135 ; 0,7615]$} & 99,13 \\
60 & 0,3380 & 4,9662 & 0,0396 & {$[-0,0177 ; 0,6937]$} & 99,32 \\
120 & 0,2970 & 4,9703 & 0,0099 & {$[0,2080 ; 0,3859]$} & 99,41 \\
180 & 0,2065 & 4,9794 & 0,0035 & {$[0,1745 ; 0,2383]$} & 99,59 \\
240 & 0,2360 & 4,9764 & 0,0170 & {$[0,0835 ; 0,3855]$} & 99,53 \\
300 & 0,1820 & 4,9818 & 0,0778 & {$[-0,5168 ; 0,8808]$} & 99,64 \\
\hline
\end{tabular}

(1) Ce: Concentração de equilíbrio

(1) qe: Capacidade de adsorção
Tabela 4 - Influência do pH da solução de cobre (II) no processo de adsorção por carvão ativado do caroço de buriti

\begin{tabular}{cccccc}
\hline pH & $\begin{array}{c}\mathrm{C}_{\mathrm{e}}{ }_{(1)} \\
\left(\mathrm{mg} \mathrm{L}^{-1}\right)\end{array}$ & $\begin{array}{c}\mathrm{q}_{\mathrm{e}}{ }^{(2)} \\
\left(\mathrm{mg} \mathrm{g}^{-1}\right)\end{array}$ & $\begin{array}{c}\text { Desvio } \\
(95 \% \text { de } \\
\text { Confiança) }\end{array}$ & $\begin{array}{c}\text { Intervalo } \\
\text { de Confiança }\end{array}$ & $\begin{array}{c}\text { Eficiência } \\
\text { de Remoção } \\
(\%)\end{array}$ \\
\hline 3,00 & 3,1460 & 4,6854 & 0,0438 & {$[2,7521 ; 3,5399]$} & 93,71 \\
4,01 & 0,3795 & 4,9621 & 0,1025 & {$[-0,5417 ; 1,3007]$} & 99,24 \\
5,18 & 0,3950 & 4,9605 & 0,0212 & {$[0,2044 ; 0,5855]$} & 99,21 \\
6,00 & 0,4485 & 4,9552 & 0,0799 & {$[-0,2694 ; 1,1664]$} & 99,10 \\
\hline
\end{tabular}

(1) Ce: Concentração de equilibrio

(2) qe: Capacidade de adsorção

\section{Influência do pH na adsorção de cobre (II)}

$\mathrm{Na}$ Tabela 4 são apresentados os resultados obtidos para a influência do pH da soluçáo inicial na adsorção de cobre (II) em CACB a $900^{\circ} \mathrm{C}$. Percebe-se que as eficiências de remoção para os quatro valores de $\mathrm{pH}$ inicial da amostra sáo bastante elevados. Contudo, a amostra a $\mathrm{pH} 3$ teve um rendimento menor quando comparada às demais. A maior quantidade de grupos carboxílicos presentes na superfície do $\mathrm{CACB}$ melhora o processo de adsorção se o $\mathrm{pH}$ de trabalho estiver situado na faixa ácida (Liu et al.2007). Terzyk (2004) estudou a variação do $\mathrm{pH}$ inicial da solução na adsorção do azul de metileno em carvão ativado de gráos de café. Ele observou que o pH inicial da solução não apresentava um efeito significativo na capacidade de adsorçáo. Como as percentagens de remoçáo foram praticamente iguais em todos os $\mathrm{pHs}$, e usando o $\mathrm{pH}$ natural se economiza reagentes, utilizou-se, entáo, o $\mathrm{pH}$ 5,18 ( $\mathrm{pH}$ natural da solução) para o ensaio de equilíbrio de adsorção.

\section{Equilíbrio de adsorção}

$\mathrm{Na}$ Tabela 5 são exibidos os resultados obtidos para a influência da concentração inicial da solução de sulfato de cobre (II) ( $\left.\mathrm{C}_{0}\right)$ na adsorção deste em CACB a $900{ }^{\circ} \mathrm{C}$. Observou-se que a eficiência de remoção de cobre (II) apresentou um máximo para as concentraçôes de 50 e $80 \mathrm{mg}$ $\mathrm{L}^{-1}$, indicando que a concentração de sulfato de cobre (II) de $50 \mathrm{mg} \mathrm{L}^{-1}$ selecionada para os ensaios anteriores foi a ideal.

Tabela 5 - Influência da concentração inicial (CO) de cobre (II) no processo de adsorção por carvão ativado do caroço de buriti

\begin{tabular}{cccccc}
\hline $\begin{array}{c}\mathrm{C}_{0} \\
\left(\mathrm{mg} \mathrm{L}^{-1}\right)\end{array}$ & $\begin{array}{c}\mathrm{C}_{\mathrm{e}}{ }^{(1)} \\
\left(\mathrm{mg} \mathrm{L}^{-1}\right)\end{array}$ & $\begin{array}{c}\mathrm{q}_{\mathrm{e}}{ }^{(2)} \\
\left(\mathrm{mg} \mathrm{g}^{-1}\right)\end{array}$ & $\begin{array}{c}\text { Desvio } \\
(95 \% \text { de } \\
\text { confiança) }\end{array}$ & $\begin{array}{c}\text { Intervalo } \\
\text { de Confiança }\end{array}$ & $\begin{array}{c}\text { Eficiência } \\
\text { de Remoção } \\
(\%)\end{array}$ \\
\hline 5 & 1,0145 & 0,3986 & 0,9200 & {$[-7,2509 ; 9,2799]$} & 79,71 \\
10 & 1,3310 & 0,8669 & 0,0509 & {$[0,8736 ; 1,7884]$} & 86,69 \\
30 & 0,4115 & 2,9589 & 0,0021 & {$[0,3924 ; 0,4306]$} & 98,63 \\
50 & 0,3685 & 4,9632 & 0,0035 & {$[0,3367 ; 0,4003]$} & 99,26 \\
80 & 0,5930 & 7,9407 & 0,0212 & {$[0,4024 ; 0,7836]$} & 99,26 \\
100 & 6,9410 & 9,3059 & 1,0126 & {$[-2,1566 ; 16,0386]$} & 93,06 \\
200 & 96,351 & 10,365 & 3,3411 & {$[66,3321 ; 126 ; 3689]$} & 51,82 \\
\hline
\end{tabular}

(1) Ce: Concentração de equilibrio

() qe: Capacidade de adsorção 
Nota-se que para concentraçôes mais baixas de cobre (II), tais como $5 \mathrm{mg} \mathrm{L}^{-1}$ e $10 \mathrm{mg} \mathrm{L}^{-1}$, as eficiências de remoção ficaram abaixo de $90 \%$. Isso se deve ao fato de se ter menores gradientes de concentração, acarretando um menor fluxo de transferência de massa nas três primeiras etapas do mecanismo de adsorçáo (Noble e Terry 2004; Bansal e Goyal 2005). Notou-se também que em concentraçóes iniciais acima de $80 \mathrm{mg} \mathrm{L}^{-1}$, inicia-se a saturação do $\mathrm{CACB}$, que é devida à saturação dos poros com cobre (II). Percebe-se que na concentração inicial de $200 \mathrm{mg}$ $\mathrm{L}^{-1}$ a eficiência é praticamente $50 \%$.

\section{Ajuste aos modelos matemáticos de equilíbrio de adsorção}

Nas Figuras 2 e 3 são apresentados os ajustes dos dados de equilíbrio para os modelos de Langmuir e Freundlich, e com os dados estatísticos para Langmuir $\left(R^{2}=0,895 ; q_{\text {máx. }}=10,289 ; b\right.$ $=2,321$ e $\left.R_{L}=0,079\right)$, e Freundlich $\left(R^{2}=0,827 ; K_{\text {ad. }}=6,186\right.$ e $\mathrm{n}=0,125)$. Observa-se que o modelo que apresentou um

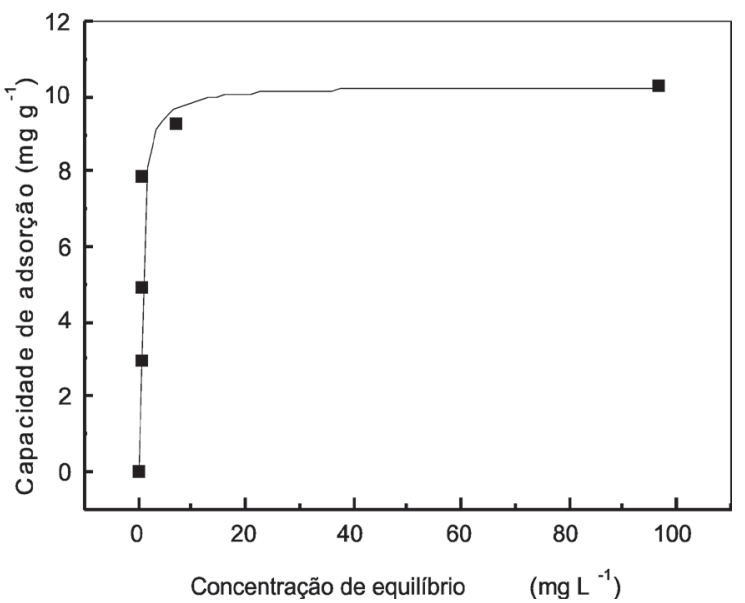

Figura 2 - Concentração de equilíbrio em função da capacidade e adsorção pelo modelo da isoterma de Langmuir

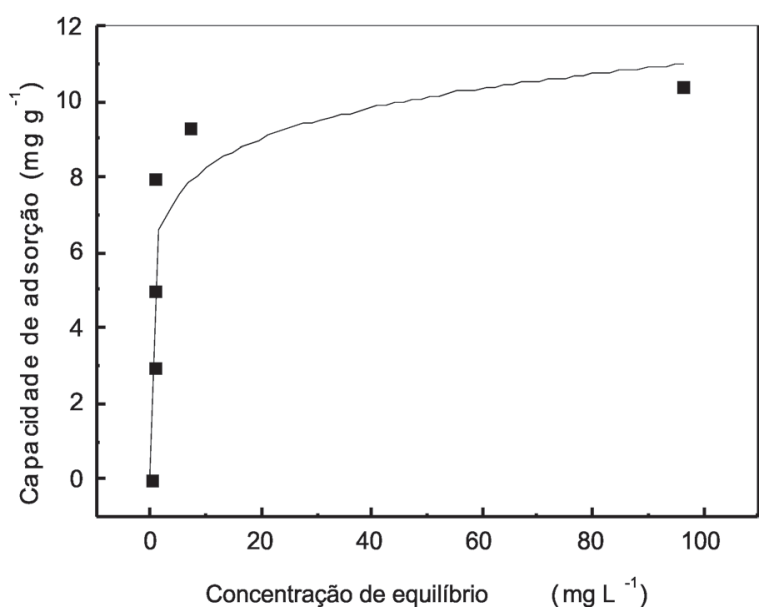

Figura 3 - Concentração de equilíbrio em função da capacidade e adsorção pelo modelo da isoterma de Freundlich bom ajuste aos dados experimentais foi o de Langmuir, devido ao maior valor do coeficiente de determinaçáo $\left(\mathrm{R}^{2}\right)$ e ao valor do fator de separação $\left(\mathrm{R}_{\mathrm{t}}\right)$ calculado, indicando adsorção do tipo favorável $\left(0<\mathrm{R}_{\mathrm{L}}<1\right)$.

\section{CONCLUSÕES}

Com base nos resultados do estudo de equilíbrio, concluise haver uma adsorção favorável, sendo mais bem descrita pelo modelo de Langmuir em comparação ao modelo de Freundlisch.

Nos resultados da eficiência de remoção obtidos nos ensaios da influência do tempo de contato adsorvente/ adsorvato na adsorção de cobre (II) observou-se que a partir do tempo de adsorção de 15 minutos, as concentraçôes de equilíbrio (Ce) da soluçáo de cobre (II) decaíram de uma concentração inicial de $50 \mathrm{mg} \mathrm{L}^{-1}$ para concentraçôes abaixo do valor máximo permitido $\left(1,0 \mathrm{mg} \mathrm{L}^{-1}\right)$ de lançamento de efluentes em corpos receptores, valor este preconizado pela legislação nacional vigente.

Observa-se nesse estudo que o CACB é um excelente adsorvente para uma soluçáo de cobre (II). Contudo, para que possamos usá-lo como adsorvente no tratamento de águas residuárias, deveremos testá-lo em efluentes industriais, in natura, que lançam elevadas cargas poluidoras em corpos receptores.

$\mathrm{O} C A C B$ pode representar uma alternativa original para os caroços de buriti, visto que eles tornam-se um rejeito comum quando o fruto é utilizado na indústria em geral, principalmente na produção de óleo comestível. Outrossim, o CACB pode representar uma soluçấo viável para os rejeitos da fabricação de biodiesel.

\section{BIBLIOGRAFIA CITADA}

Aguiar, M.R.M.P.; Novaes, A.C. 2002. Removal of heavy metals from wastewaters by aluminosilicate. Química Nova, 25 (6B), $1145-1154$

Ajmal, M.; Rao, R.A.K.; Khan, M.A. 2005. Adsorption of copper from aqueous solution on Brassica cumprestris (mustard oil cake). Journal of Hazardous Material, 122 (1-2), 177-183.

Banat, F.; Al-Asheh, S.; Al-Makhadmeh, L. 2003. Evaluation of the use of raw and activated date pits as potential adsorbents for dye containing waters. Process Biochemistry, 39 (2): 193-202.

Bansal, R.C.; Goyal, M. Activated Carbon Adsorption. 2005. New York: Taylor \& Francis. 497 pp.

Beck, N.V.; Meech, S.E.; Norman, P.R.; Pears, L.A. 2002. Characterization of surface oxides on carbon and their influence on dynamic adsorption. Carbon, 40: 531-540.

Boehm, H. P. 1994. Some aspect of the surface chemistry of carbon blacks and other carbons. Carbon, 32: 759-769.

Boehm, H. P. 2002. Surface oxides on carbon and their analysis: a critical assessment. Carbon, 40: 145-149. 
Braile, P.M.; Cavalcanti, J.E.W.A. 1993. Industrial Wastewater Treatment Handbook. São Paulo: CETESB, 764 pp (in Portuguese).

Brasil. 2011. Resolução do Conselho Nacional do Meio Ambiente, número 430, publicada em 13 de maio de 2011. Diário Oficial da República Federativa do Brasil, Brasília, DF. 9 pp.

Bryan, G.W.; Langston, W.J. 1992. Bioavailability, accumulation and effects of heavy metals in sediments with special reference to United Kingdom estuaries: a review. Environmental Pollution, 76: 89-131.

Crini, G. 2006. Non-conventional low-cost adsorbents for dye removal: a review. Bioresource Technology, 97: 1061-1085.

Di Bernardo, L.; Dantas, A. Di B. 2005. 1992. Methods and Techniques of Water Treatment. 2. ed. São Carlos: RiMa. 2: 1495-1535 (in Portuguese).

Kadirvelu, K.; Thamaraiselvi, K.; Namasivayam, C. 2001. Adsorption of nickel (II) from aqueous solution onto activated carbon prepared from coirpith. Separation and Purification Technology, 24: 497-505.

Kaikake, K.; Hoaki, K.; Sunada, H.; Dhakal, R.P.; Baba, Y. 2007. Removal characteristics of metal ions using degreased coffee beans: Adsorption equilibrium of cadmium (II). Bioresource Technology, 98: 2787-2791.

Karagöz, S.; Tay, T.; Ucar, S.; Erdem, M. 2008. Activated carbons from waste biomass by sulfuric acid activation and their use on methylene blue adsorption. Bioresource Technology, 99: 6214-6222.

Lee, J.D. 1980. Inorganic Chemistry: a new concise text. São Paulo: Edgard Blücher. 374-382 (in Portuguese).

Liu, S.X.; Chen, X.; Liu, Z.F.; Wang, H.L. 2007. Activated carbon with excellent chromium (VI) adsorption performance prepared by acid-base surface modification. Journal of Hazardous Material, 141: 315-319.

Manhães, L.R.T. 2007. Buriti pulp characterization (Mauritia flexuosa Mart): A powerful functional food. Rio de Janeiro, 2007. Dissertação (Mestrado) Universidade Federal Rural do Rio de Janeiro. Instituto de Tecnologia. 187 pp (in Portuguese).
Moreno-Castilla, C. 2004. Adsorption of organic molecules from aqueous solution on carbon materials. Carbon, 42: 83-94.

Noble, R.D.; Terry, P.A. 2004. Principles of Chemical Separations with Environmental Application. Cambridge, UK: Cambridge University Press, 182-213.

Nunes, A.A.; França, A.S.; Oliveira, L.S. 2009. Activated carbons from waste biomass: An alternative use for biodiesel production solid residues. Bioresource Technology, 100(5): 1705-1884.

Ramos, M.A.B. 2005. Cr (III) ion removal from aqueous solution by adsorption on activated carbon and biosorption and immobilized yeast. Belém, 2005. Dissertação (Mestrado) Universidade Federal do Pará. 112 pp (in Portuguese).

Senthilkumaar, S.; Varadarajan, P.R.; Porkodi, K.; Subbhuraam, C.V. 2005. Adsorption of methylene blue onto jute fiber carbon: kinetics and equilibrium studies. Journal of Colloid and Interface Science, 284: 78-825.

Singh, K.P.; Moham, D.; Sinha, S.; Tondon, G.S.; Gosh, D. 2003. Collor removal from wastewater using low-cost activated carbon derived from agricultural waste material. Industrial and Engineering Chemistry Research, 42 (9): 1965-1976.

Souza, R.S. 2009. Chromium (VI) adsorption by granular activated carbon from diluted solutions using a batch system under controlled pH. Acta Amazonica, 39(3): 661-668 (in Portuguese, with abstract in English).

Tan, I.A.W.; Hameed, B.H.; Ahmad, A.L. 2007. Equilibrium and kinetic studies on basic dye adsorption by oil palm fibre activated carbon. Chemical Engineering Journal, 127: 111-119.

Terzyk, A.P. 2004. Molecular properties and intermolecular forcesfactors balancing the effect of carbon surface chemistry in adsorption of organics from diluted aqueous solution. Journal of Colloid and Interface Science, 275: 9-29.

Zhao, N.; Wei N.; Li, J.; Qiao, Z.; Cui. J.; He, F. 2005. Surface properties of chemically modified activated carbons for adsorption rate of $\mathrm{Cr}(\mathrm{VI})$. Chemical Engineering Journal, 115: 133-138.

Recebido em: 27/09/2011

Aceito em: 30/01/2012 